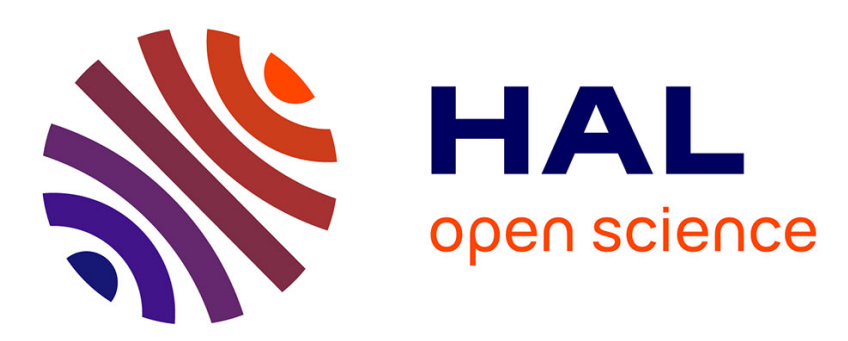

\title{
Output feedback transient stabilization and voltage regulation of synchronous generators
}

\author{
Cristiano Maria Verrelli, G. Damm
}

\section{To cite this version:}

Cristiano Maria Verrelli, G. Damm. Output feedback transient stabilization and voltage regulation of synchronous generators. International Journal of Robust and Nonlinear Control, 2012, 22 (13), pp.1495-1504. 10.1002/rnc.1762 . hal-02936784

\section{HAL Id: hal-02936784 \\ https://hal.science/hal-02936784}

Submitted on 11 Sep 2020

HAL is a multi-disciplinary open access archive for the deposit and dissemination of scientific research documents, whether they are published or not. The documents may come from teaching and research institutions in France or abroad, or from public or private research centers.
L'archive ouverte pluridisciplinaire HAL, est destinée au dépôt et à la diffusion de documents scientifiques de niveau recherche, publiés ou non, émanant des établissements d'enseignement et de recherche français ou étrangers, des laboratoires publics ou privés. 


\title{
Output Feedback Transient Stabilization and Voltage Regulation of Synchronous Generators
}

\author{
Cristiano Maria Verrelli*, Gilney Damm** \\ *Dipartimento di Ingegneria Elettronica, Universitá di Roma "Tor Vergata" \\ Via del Politecnico 1, 00133 Rome, Italy \\ E-mail: verrelli@ing.uniroma2.it \\ **Laboratoire des Signaux et Systèmes, L2S-CNRS \\ Plateau de Moulon, Gif sur Yvette 91192, France \\ E-mail: gilney.damm@lss.supelec.fr
}

\begin{abstract}
In this brief note we consider the transient stabilization and voltage regulation problem addressed in Ref. (1) for a synchronous generator connected to an infinite bus. We show how a much simpler output feedback controller can be obtained if a different stability analysis is carried out: a suitable change of coordinates is performed so that advantageous triangularity properties of the regulation error dynamics are highlighted. A novel first order estimator for the uncertain mechanical input power is designed which, in contrast to Ref. (1), is the only adaptation scheme used by the controller. The innovative crucial step in the presented control design relies on relaxing the constraint imposed by the backstepping techniques adopted in Ref. (1) and constituted by the required availability of the regulation/tracking error variables: the converging estimate of the mechanical input power directly becomes the reference value for the electrical power and allows for the straightforward computation of the uncertain power angle constant value guaranteeing voltage regulation at steady-state.
\end{abstract}

\section{Introduction}

The control of synchronous generators constitutes a very important and challenging topic in the power systems operation field: transient stability is to be enhanced and terminal voltage is to be regulated to its reference value in spite of sudden mechanical and electrical perturbations which may permanently change the power network structure. Nonlinear controllers, in contrast to the classical Power System Stabilizer/Automatic Voltage Regulator schemes, are not designed on the basis of linearizations around operating conditions but incorporate nonlinear feedback terms which allow for enlarging the machine stability region. Their structures can be however rather complicated - with consequent implementation difficulties - especially when the transient stabilization and voltage regulation problems are simultaneously solved in the presence of system parameter uncertainties: among the most relevant results, see Ref. (2) for a state feedback solution ${ }^{1}$ in the presence of uncertainties in almost all system parameters and Ref. (1) for an output feedback solution in the presence of transmission line impedance and mechanical power uncertainties.

In this paper we show how a nonlinear adaptive output feedback controller, which is much simpler than the one presented in Ref. (1), solves the same problem addressed in Ref. (1). A stability analysis which involves an advantageous change of coordinates is carried out: it highlights the triangularity properties of the regulation error

\footnotetext{
${ }^{1}$ Theoretical foundations can be found in Ref. (3).
} 
dynamics so that the constraint, imposed by the back-stepping techniques adopted in Ref. (1) (see also Refs. $(2 ; 4 ; 5 ; 6)$ for similar approaches) and constituted by the required availability of the regulation/tracking error variables, is definitely removed. The gap between simple structure of the controller and large stability regions of the machine is thus filled: the new controller is a simple feedback linearizing controller which only incorporates a reduced order estimator for the uncertain mechanical input power and does not rely on the knowledge of the transmission line impedance and on the measurements of the power angle (both computed, as in Ref. (1), via static formulas). In contrast to the strategy adopted by the classical PSS/AVR scheme (see Refs. $(7 ; 8)$ ) which considers the terminal voltage as the natural controlled output (with minimum phase characteristics), the strategy adopted in this paper (as well as in Refs. $(1 ; 2 ; 4))$ transforms the power angle in the controlled output by means of the inversion formula derived in Ref. (1). The resulting control law is, at the same time, a feedback linearizing and an input-output linearizing control in which the converging estimate of the mechanical input power directly constitutes the reference value for the electrical power and allows for the straightforward computation of the uncertain power angle constant value guaranteeing voltage regulation at steady-state.

\section{Problem statement}

The third order dynamic model of a synchronous generator connected to an infinite bus (see Ref. (1) for its description and Ref. (6) for a more general model) is given by

$$
\begin{aligned}
\dot{\delta} & =\omega \\
\dot{\omega} & =-\frac{D}{H} \omega-\frac{\omega_{s}}{H} P_{e}+\frac{\omega_{s}}{H} P_{m} \\
\dot{P}_{e} & =-\frac{1}{T_{d 0}^{\prime}} P_{e}+\frac{\left(X_{d}-X_{d}^{\prime}\right)}{X_{d s} X_{d s}^{\prime}} V_{s}^{2} \omega \sin ^{2} \delta+P_{e} \omega \operatorname{cotg} \delta+\frac{V_{s} K_{c}}{X_{d s} T_{d 0}^{\prime}}(\sin \delta) u_{f}
\end{aligned}
$$

in which: $\delta(\mathrm{rad})$ denotes the generator power angle with respect to the infinite bus rotating at synchronous speed $\omega_{s} ; \omega=\omega_{g}-\omega_{s}(\mathrm{rad} / \mathrm{s})$ is the difference between the generator angular speed $\omega_{g}$ and $\omega_{s} ; P_{e}(p . u$.$) denotes the active electrical power delivered by$ the generator to the infinite bus; $u_{f}(p . u$. $)$ is the input to the SCR amplifier of the generator; $P_{m}(p . u$. $)$ is the mechanical input power; $H(s)$ is the inertia constant, $D(p . u$.$) is$ the damping constant and $P_{m}\left(p . u\right.$.) is the mechanical input power; $V_{s}(p . u$.) is the voltage at the infinite bus; $X_{d s}=X_{s}+X_{d} \doteq X_{T}+\frac{1}{2} X_{L}+X_{d}(p . u$.) is the total reactance which takes into account the generator direct axis reactance $X_{d}(p . u$. $)$, the transmission line reactance $X_{L}(p . u$.$) and the transformer reactance X_{T}(p . u.) ; X_{d s}^{\prime}(p . u$.$) is equal to$ $X_{s}+X_{d}^{\prime}$ with $X_{d}^{\prime}$ denoting the generator direct axis transient reactance; $K_{c}$ is the gain of the excitation amplifier and $T_{d 0}^{\prime}$ is equal to $T_{d 0} X_{d s}^{\prime} / X_{d s}$ with $T_{d 0}(s)$ being the direct axis short circuit time constant. The variables $\left(\delta, \omega, P_{e}\right)$ constitute the states of model (1) while $u_{f}$ is the control signal to be designed. The generator terminal voltage is given by

$$
V_{t}=\left[\frac{X_{s}^{2} P_{e}^{2}}{V_{s}^{2} \sin ^{2} \delta}+\frac{X_{d}^{2} V_{s}^{2}}{X_{d s}^{2}}+\frac{2 X_{s} X_{d}}{X_{d s}} P_{e} \operatorname{cotg} \delta\right]^{\frac{1}{2}}
$$

which is to be regulated to its reference value $V_{t r}=1(p . u$.) (voltage regulation) while the relative speed $\omega$ is to be kept close and regulated to zero (transient stabilization). 
In order to obtain a more compact notation, we use the reparameterized model

$$
\begin{aligned}
\dot{\delta} & =\omega \\
\dot{\omega} & =-\frac{D}{H} \omega-\frac{\omega_{s}}{H} P_{e}+\frac{\omega_{s}}{H} P_{m} \\
\dot{P}_{e} & =-\theta_{1} P_{e}+\theta_{2} \omega \sin ^{2} \delta+P_{e} \omega \operatorname{cotg} \delta+\theta_{3}(\sin \delta) u_{f}
\end{aligned}
$$

where the three positive parameters $\theta_{i}>0,1 \leq i \leq 3$, are defined as

$$
\theta_{1}=\frac{1}{T_{d 0}^{\prime}}, \quad \theta_{2}=\frac{\left(X_{d}-X_{d}^{\prime}\right)}{X_{d s} X_{d s}^{\prime}} V_{s}^{2}, \quad \theta_{3}=\frac{V_{s} K_{c}}{X_{d s} T_{d 0}^{\prime}} .
$$

In order to avoid the physical singularities at $\delta=0$ and $\delta=\pi$, we have to restrict the operation of the system to an arbitrarily large compact set $\mathcal{B}$ contained in the open set

$$
\mathcal{D}=\left\{\left(\delta, \omega, P_{e}\right):\left(\delta, \omega, P_{e}\right) \in(0, \pi) \times \mathbb{R} \times \mathbb{R}^{+}\right\} .
$$

The asymptotically stable operating condition is given by

$$
\delta=\delta_{s}, \quad \omega=0, \quad P_{e}=P_{m}
$$

where (see Ref. (1))

$$
\delta_{s}=\operatorname{arccotg}\left[-\frac{V_{s}^{2} X_{d}}{X_{s} P_{m}\left(X_{s}+X_{d}\right)}+\sqrt{\frac{V_{s}^{2} V_{t r}^{2}}{X_{s}^{2} P_{m}^{2}}-1}\right] \in\left(0, \frac{\pi}{2}\right)
$$

guarantees terminal voltage regulation $\left[V_{t}=V_{t r}\right]$. Note that, according to the formula (see Ref. (2))

$$
\delta=\operatorname{arccotg}\left[-\frac{V_{s}^{2} X_{d}}{X_{s} P_{e}\left(X_{s}+X_{d}\right)} \pm \sqrt{\frac{V_{s}^{2} V_{t}^{2}}{X_{s}^{2} P_{e}^{2}}-1}\right]
$$

there is another value $\delta_{u} \in\left[\frac{\pi}{2}, \pi\right)$ for $\delta$ which also guarantees terminal voltage regulation and corresponds to an unstable equilibrium for system (1) fed by the open loop control

$$
u_{f u}=\frac{\theta_{1} P_{m}}{\theta_{3} \sin \delta_{u}}
$$

As remarked in Ref. (1), the mechanical input power and the transmission line impedance are to be necessarily considered, in the control design, as uncertain parameters: the mechanical input power may abruptly change to a faulted value in the case of turbine failures (mechanical perturbations) while $X_{s}$, which is the impedance of the transmission line up to the point of the power network in which the voltage is equal to $V_{s}$, may undergo sudden variations when faults on the transmission line occur (electrical perturbations). Furthermore, the power angle $\delta$ is not simply measurable and assuming its availability in the control design makes rather difficult the implementation of the resulting controller. Accordingly, while the values of $X_{s}$ and $\delta$ 
are computed in this paper through the following formulas derived in Ref. (1) ( $Q$ is the reactive power)

$$
\begin{aligned}
\delta & =\operatorname{arccotg}\left[-\frac{V_{s}^{2} X_{d}}{X_{s} P_{e}\left(X_{s}+X_{d}\right)} \pm \sqrt{\frac{V_{s}^{2} V_{t}^{2}}{X_{s}^{2} P_{e}^{2}}-1}\right] \\
X_{s} & =\frac{-Q V_{s}^{2} \pm \sqrt{Q^{2} V_{s}^{4}-\left(Q^{2}+P_{e}^{2}\right)\left(V_{s}^{2}-V_{t}^{2}\right) V_{s}^{2}}}{Q^{2}+P_{e}^{2}}
\end{aligned}
$$

the mechanical input power (assumed to be constant), as in Ref. (6), is exponentially estimated by the first order estimator $\left(\gamma_{p}\right.$ is an arbitrary positive design parameter while $\xi_{p}(0)$ is chosen so that $\left.\hat{P}_{m}(0)>0\right)$

$$
\begin{aligned}
\hat{P}_{m} & =\gamma_{p} \frac{H}{\omega_{s}} \omega+\xi_{p} \\
\dot{\xi}_{p} & =\gamma_{p} P_{e}-\gamma_{p} \xi_{p}-\gamma_{p}^{2} \frac{H}{\omega_{s}} \omega+\gamma_{p} \frac{D}{\omega_{s}} \omega
\end{aligned}
$$

which, as we shall see, in contrast to Ref. (1) will be the only adaptation scheme used by the controller. If we define the mechanical power estimation error $\tilde{P}_{m}=P_{m}-\hat{P}_{m}$, by straightforward computations we obtain

$$
\dot{\tilde{P}}_{m}=-\gamma_{p} \tilde{P}_{m}
$$

the estimation error $\tilde{P}_{m}(t)$ thus exponentially decays to zero with an arbitrarily large exponential rate of convergence. As we shall see, this converging estimate $\hat{P}_{m}$ will constitute the reference value for the electrical power $P_{e}$ and will allow for the straightforward computation of the uncertain power angle constant value $\delta_{s}$ guaranteeing voltage regulation at steady-state. Define, in fact, the regulation errors $\tilde{\delta}=\delta-\delta_{s}, \tilde{\omega}=\omega$, $\tilde{P}_{e}=P_{e}-P_{m}$ so that we can write

$$
\begin{aligned}
\dot{\tilde{\delta}} & =\tilde{\omega} \\
\dot{\tilde{\omega}} & =-\frac{D}{H} \tilde{\omega}-\frac{\omega_{s}}{H} \tilde{P}_{e} \\
\dot{\tilde{P}}_{e} & =-\theta_{1} P_{e}+\theta_{2} \omega \sin ^{2} \delta+P_{e} \omega \operatorname{cotg} \delta+\theta_{3}(\sin \delta) u_{f}
\end{aligned}
$$

In contrast to the back-stepping techniques adopted in Ref. (1) (see also Refs. (2; 4; 5 ; 6) for similar approaches), the constraint constituted by the required availability of the regulation/tracking error variables will be definitely removed so that a much simpler controller will be obtained ${ }^{2}$. On the other hand, in contrast to the strategy adopted by the classical PSS/AVR scheme which considers the terminal voltage as the natural controlled output (with minimum phase characteristics), the strategy which will be adopted in this paper (as well as in Refs. $(1 ; 2 ; 4)$ ) will transform, by means of the inversion formula (2), the power angle in the output to be controlled. To this purpose, define the feedback linearizing and input-output linearizing control

$$
u_{f}=\frac{1}{\theta_{3}(\sin \delta)}\left[\theta_{1} P_{e}-\theta_{2} \omega \sin ^{2} \delta-P_{e} \omega \operatorname{cotg} \delta+v\right]
$$

\footnotetext{
${ }^{2}$ It is however worth noting that the two procedures are, in practice, not totally equivalent (see for instance the effect of a time-varying perturbation on the mechanical input power on the regulation errors $\left(z_{1}, z_{2}, z_{3}\right)$ which will be introduced in the following).
} 
where $v$ is the additional control input yet to be designed, so that the regulation error dynamics

$$
\begin{aligned}
\dot{\tilde{\delta}} & =\tilde{\omega} \\
\dot{\tilde{\omega}} & =-\frac{D}{H} \tilde{\omega}-\frac{\omega_{s}}{H} \tilde{P}_{e} \\
\dot{\tilde{P}}_{e} & =v
\end{aligned}
$$

are linear and input-output linearized with respect to the controlled output $\tilde{\delta}$ and the new input $v$. We now introduce a suitable change of coordinates with the aim of highlighting advantageous triangularity properties of the regulation error dynamics. If we perform the change of coordinates

$$
\begin{aligned}
z_{1} & =\tilde{\delta} \\
z_{2} & =\tilde{\omega} \\
z_{3} & =-\frac{D}{H} \tilde{\omega}-\frac{\omega_{s}}{H} \tilde{P}_{e}
\end{aligned}
$$

then we obtain the triangular linear system

$$
\begin{aligned}
& \dot{z}_{1}=z_{2} \\
& \dot{z}_{2}=z_{3} \\
& \dot{z}_{3}=-\frac{D}{H} z_{3}-\frac{\omega_{s}}{H} v .
\end{aligned}
$$

It is clear that, if the error variables $z_{1}, z_{2}, z_{3}$ were available for feedback, then the virtual control

$$
v=-\frac{H}{\omega_{s}}\left[-k_{1} z_{1}-k_{2} z_{2}-\left(k_{3}-\frac{D}{H}\right) z_{3}\right]
$$

would guarantee exponential transient stabilization and voltage regulation (with arbitrarily large exponential rate of convergence $)^{3}$ : the arbitrary positive constant control parameters $k_{1}, k_{2}, k_{3}$ are to be chosen in order to assign suitable Hurwitz roots to the polynomial $s^{3}+\sum_{i=3}^{1} k_{i} s^{i-1}$ and thus to impose satisfactory transient behaviours to the regulation error dynamics. Both the error variables $z_{1}$ and $z_{3}$ are however not measurable since they depend on the uncertain $P_{m}$ (which, as in Ref. (1), will be reasonably assumed to belong to the known compact set $\left[P_{m, m}, P_{m, M}\right]$ with $\left.P_{m, M}>P_{m, m}>0\right)$. Since a converging estimate for $P_{m}$ is provided by the estimator (5), we thus modify the virtual input $v$ into the implementable one

$$
\begin{aligned}
v & =-\frac{H}{\omega_{s}}\left[-k_{1} \hat{z}_{1}-k_{2} z_{2}-\left(k_{3}-\frac{D}{H}\right) \hat{z}_{3}\right] \\
& =-\frac{H}{\omega_{s}}\left[-k_{1} \hat{z}_{1}-k_{2} \tilde{\omega}-\left(k_{3}-\frac{D}{H}\right) \hat{z}_{3}\right]
\end{aligned}
$$

\footnotetext{
${ }^{3}$ According to the definition of $z_{1}, z_{2}, z_{3}$, guaranteeing their exponential convergence to zero implies guaranteeing exponential convergence to zero of the regulation errors $\tilde{\delta}(t), \tilde{\omega}(t)$, $\tilde{P}_{e}(t)$ (so that transient stabilization is achieved). On the other hand, since $\left(N_{v \delta}, N_{v p}\right.$ are suitable positive reals)

$$
\left|V_{t}-V_{t r}\right| \leq N_{v \delta}|\tilde{\delta}|+N_{v p}\left|\tilde{P}_{e}\right|
$$

for any trajectory belonging to $\mathcal{B}$ and for any $t \geq 0$, exponential voltage regulation is guaranteed.
} 
where the estimated variables $\hat{z}_{1}$ and $\hat{z}_{3}$ are intuitively chosen as

$$
\begin{aligned}
& \hat{z}_{1}=\delta-\hat{\delta}_{s} \\
& \hat{z}_{3}=-\frac{D}{H} \tilde{\omega}-\frac{\omega_{s}}{H}\left(P_{e}-\hat{P}_{m}^{s}\right)
\end{aligned}
$$

with $^{4}$

$$
\begin{aligned}
\hat{\delta}_{s} & =\operatorname{arccotg}\left[-\frac{V_{s}^{2} X_{d}}{X_{s} \hat{P}_{m}^{s}\left(X_{s}+X_{d}\right)}+\sqrt{\frac{V_{s}^{2} V_{t r}{ }^{2}}{X_{s}{ }^{2}\left(\hat{P}_{m}^{s}\right)^{2}}-1}\right] \\
\hat{P}_{m}^{s} & =\operatorname{sat}\left(\hat{P}_{m}\right) .
\end{aligned}
$$

In this way, as announced the converging estimate $\hat{P}_{m}$ of the mechanical input power not only constitutes the reference value for the electrical power $P_{e}$ but it also allows for the straightforward computation of the uncertain power angle constant value $\delta_{s}$ guaranteeing voltage regulation at steady-state. The closed loop system can be consequently written as

$$
\begin{aligned}
& \dot{z}_{1}=z_{2} \\
& \dot{z}_{2}=z_{3} \\
& \dot{z}_{3}=-k_{1} z_{1}-k_{2} z_{2}-k_{3} z_{3}+h\left(t, P_{m}-\hat{P}_{m}^{s}\right)
\end{aligned}
$$

where $h\left(t, P_{m}-\hat{P}_{m}^{s}\right)$ is a suitable continuous function which satisfies ( $N_{h}$ is a suitable positive real)

$$
\left|h\left(t, P_{m}-\hat{P}_{m}^{s}\right)\right| \leq N_{h}\left|P_{m}-\hat{P}_{m}^{s}\right|, \quad \forall t \geq 0 .
$$

Since the estimation error $\tilde{P}_{m}(t)$ exponentially decays to zero with an arbitrarily large exponential rate of convergence, by virtue of the Lemma A.4 in Ref. (9), we can establish that transient stabilization and exponential voltage regulation are achieved (with an arbitrarily large exponential rate of convergence) for restricted generator initial conditions which keep the state variables inside the admissible set $\mathcal{B}$ for any time $t \geq 0$. In conclusion, the resulting controller (3)-(9), which is apparently much simpler than the one presented in Ref. (1), is able to solve the same problem addressed in Ref. (1).

\section{Conclusions}

The transient stabilization and voltage regulation problem addressed in Ref. (1) for a synchronous generator connected to an infinite bus is considered. A much simpler output feedback controller is presented in which the converging estimate of the mechanical input power directly constitutes the reference value for the electrical power and allows for the straightforward computation of the uncertain power angle constant value guaranteeing voltage regulation at steady-state.

\footnotetext{
${ }^{4}$ Even though its introduction is not necessary for the stability analysis, we use, for robustness issues, the saturation function $\operatorname{sat}(x)$ which is equal to: i) $x$ in the compact set $\left[P_{m, m}, P_{m, M}\right]$; ii) $P_{m, m}$ for $x \leq P_{m, m}$; iii) $P_{m, M}$ for $x \geq P_{m, M}$.
} 


\section{Acknowledgements}

The first author is indebted to Prof. R. Marino for helpful discussions concerning this article.

\section{References}

[1] G. Damm, R. Marino, and F. Lamnabhi-Lagarrigue, Adaptive nonlinear output feedback for transient stabilization and voltage regulation of power generators with unknown parameters, International Journal of Robust and Nonlinear Control, 14: 833-855, 2004.

[2] G. Damm, F. Lamnabhi-Lagarrigue, R. Marino, and C.M. Verrelli, Transient stabilization and voltage regulation of a synchronous generator, in Taming Heterogeneity and Complexity of Embedded Control, Editors: F. Lamnabhi-Lagarrigue, S. Laghrouche, A. Loria and E. Panteley, International Scientific \& Technical Encyclopedia (ISTE), London, 211-225, 2007.

[3] R. Marino, and C.M. Verrelli, A global state feedback output regulating control for uncertain systems in strict feedback form, Systems and Control Letters, 58: 682-690, 2009.

[4] X. Jiao, Y. Sun, and T. Shen, Adaptive controller design for a synchronous generator with unknown perturbation in mechanical power, International Journal of Control, Automation, and Systems, 3: 308-314, 2005.

[5] R. Marino, T. Shen, and C.M. Verrelli, Robust adaptive transient stabilization of a synchronous generator with parameter uncertainty, European Journal of Control, 12: 135-155, 2006.

[6] C.M. Verrelli, and G. Damm, Robust transient stabilization problem for a synchronous generator in a power network, International Journal of Control, 83: 816-828, 2010.

[7] H. Bourlés, S. Peres, T. Margotin, and M.P. Houri, Analysis and design of a robust coordinated AVR/PSS, IEEE Transactions on Power Systems, 13: 568-575, 1998.

[8] H. Quinot, H. Bourlés, and T. Margotin, Robust coordinated AVR+PSS for damping large scale power systems, IEEE Transactions on Power Systems, 14: 1446-1451, 1999.

[9] R. Marino, P. Tomei, and C.M. Verrelli, Induction Motor Control Design, Springer, 2010. 